\title{
Zur ambivalenten Geschichte der deutschen Amtssprache
}

\author{
Sarah Praunsmändel
}

\begin{abstract}
Anhand der Geschichte der deutschen Amtssprache, der ibr zugrunde liegenden Sprachenpolitik und den diese leitenden zeitgeschichtlichen Ideen und Kräften offenbart sich die grundsätzliche Ambivalenz des Sprachenrechts: Die deutsche Amtssprache hat je nach zeitgeschichtlichem Kontext entweder den Zugang zu Recht gewährt oder verhindert. Im historischen Großbild, beginnend mit der Gegenwart und zurückgehend bis in das Heilige Römische Reich, zeigt sich, dass der im Deutschen Kaiserreich aufkommende Nationalismus, der die notwendige Einheit von Volk, Nation und Sprache postulierte, den Wendepunkt hin $z u$ gruppenspezifischen Amtssprachenregelungen markiert. Auch \23 Abs. 1 VwVfG, der 1973 aufgrund der Sprachenprobleme der Verwaltung im Angesicht zahlreicher "Gastarbeiter" eingeführt wurde, lässt sich in seinem Entstehungskontext als gruppenspezifische Regelung begreifen.
\end{abstract}

\section{Einfübrung}

Sprechen zu dürfen und gehört zu werden ${ }^{1}$ sind zentrale Voraussetzungen für den Zugang zu Recht. Hieraus folgt, dass Sprachbarrieren in rechtsstaatlichen Verfahren keine Hindernisse sein dürfen. ${ }^{2}$ Für die Gerichtsbarkeit bestimmt $\mathbb{1} 184$ S. 1 GVG: „Die Gerichtssprache ist deutsch“, ergänzend legen die $\mathbb{S} 185 \mathrm{ff}$. GVG umfassende Übersetzungspflichten fest.

Auch wenn der Wortlaut des $\$ 184$ S. 1 GVG dem Wortlaut des $\$ 23$ Abs. 1 VwVfG („Die Amtssprache ist deutsch“) zwillingshaft ähnelt, können wir nicht von einem vergleichbaren Schutzniveau für deutschunkundige Verfahrensbeteiligte im Verwaltungsverfahren sprechen. Denn

1 Remmert, in: Maunz/Dürig, Grundgesetz-Kommentar, 92. EL August 2020, Art. 103 Rn. 60 f. u. 92 f., gängig formuliert als Äußerungsrecht und das Recht auf Berücksichtigung.

2 Das Recht auf Zuziehung eines/r Dolmetschers/in im Gerichtsverfahren ergibt sich für das Zivilverfahren wohl direkt aus Art. 103 Abs. 1 GG, speist sich aber auch aus dem Grundrecht auf ein faires Verfahren: Remmert, GG (Fn. 1), Art. 103 Rn. 31, insbes. Fn. 2. 
obwohl auch im Verwaltungsverfahren das Recht auf ein faires Verfahren ${ }^{3}$ und das Recht auf Gehör gelten, ${ }^{4}$ kennt es, anders als das gerichtliche Verfahren, keine generelle staatliche Übersetzungspflicht. ${ }^{5}$ Diese monolinguale Ausrichtung des Verwaltungsverfahrens trägt Geschichte in sich.

In einem ersten Schritt soll es um die Entstehungsgeschichte des $\$ 23$ VwVfG gehen, bevor die historische Entwicklung der deutschen Amtssprache beginnend mit dem Heiligen Römischen Reich zurückverfolgt wird. Dabei gilt der Blick mehr den großen ideengeschichtlichen Entwicklungsbögen, die verändernden Einfluss auf die Amtssprache hatten, als einer vollständigen rechtshistorischen Abbildung. Dieser rechtsgeschichtliche Ansatz will also weniger die Entstehung der Amtssprache nachzeichnen, als vielmehr den Versuch unternehmen, die Ursachen und Kräfte, die Gedanken und geistigen Strömungen aufzudecken, die die Entwicklung der Rechtsidee (deutsche) Amtssprache beeinflusst haben und vor allem: noch im Gegenwartsrecht fortwirken. ${ }^{6}$

\section{Die Entstehung der Amtssprachenregelung im VwVfG}

Eine Amtssprachenregelung ist den Vorläufern des VwVfG fremd. Sie findet sich erstmals im Regierungsentwurf von 1973. ${ }^{7}$ Auch die nach 1945

3 Schoch, in: Schoch/Schneider (Hrsg.), VwVfG, Stand: Juli 2020, Einleitung Rn. 300.

4 Kallerhoff/Mayen, in: Stelkens/Bonk/Sachs, VwVfG, 9. Aufl. 2018, \$28 Rn. 2; Schoch, in: Schoch/Schneider VwVfG (Fn. 3), Einleitung Rn. 328.

5 Rixen, in: Schoch/Schneider VwVfG (Fn. 3), $\$ 23$ Rn. 24; Ziekow, VwVfG, 4. Aufl. 2019, $\mathbb{2} 23$ Rn. 6; Schwarz, in: Fehling/Kastner/Störmer (Hrsg.), Verwaltungsrecht, $\$ 23$ VwVfG Rn. 4; für eine teilweise Übersetzungspflicht: Kuntze/Beichel-Benedetti, in: Obermayer/Funke-Kaiser (Hrsg.), VwVfG, 5. Aufl. 2018, $\$ 23$ Rn. 56 ff.; eine generelle Übersetzungspflicht ebenfalls ablehnend Schmitz, in: Stelkens/Bonk/Sachs VwVfG (Fn. 4), $\mathbb{} 23$ Rn. 41.

6 Conrad, Deutsche Rechtsgeschichte, Bd. I, 2. Aufl. 1962, XVII (Einleitung).

7 Stelkens, P./Schmitz, in: Stelkens/Bonk/Sachs, VwVfG, 6. Aufl. 2001, \$23 Entstehungsgeschichte; BT-Drucks. 7/910, S. 12. 
eingeführten Landesgesetze ${ }^{8}$ und die Gesetzesentwürfe ${ }^{9}$ zur Kodifikation des Verwaltungsverfahrens auf Länderebene enthielten keine Regelungen zur Amtssprache. Ebenso wenig sahen der erste Musterentwurf eines Verwaltungsverfahrensgesetzes des Bundes von 1963 oder die „Münchener Fassung" des Musterentwurfs von $1966^{10}$, noch der erste Regierungsentwurf eines Verwaltungsverfahrensgesetzes von $1970^{11}$ eine entsprechende Regelung vor.

\section{1. „Ist für Ausländer die Verwaltungssprache deutsch?“}

Die Aufnahme der Regelung war das Ergebnis einer erst kurz zuvor begonnenen Debatte. 1971 erscheint unter dem Titel „Ist für Ausländer die Verwaltungssprache deutsch?" ein Aufsatz von Hans-Christian von Ebner, ${ }^{12}$ der die Notwendigkeit einer Amtssprache erstmals proklamierte. Der Regierungsdirektor beginnt seine Ausführungen so:

„Im vergangenen Jahr lebten beinahe 3 Millionen Ausländer, vor allem Gastarbeiter, in der Bundesrepublik; mehr als 7,5 Millionen Ausländer haben außerdem im letzten Jahr die zweitgrößte Handelsnation der Welt besucht. (...) in erster Linie die Verwaltung muss sich daher in zunehmendem Maße mit Angelegenheiten befassen, an denen Ausländer beteiligt sind. Dabei treten nicht selten Verständigungsschwierigkeiten auf $(\ldots)$ “13

8 Vgl. Verwaltungsverfahrensgesetz des Landes Bremen v. 11.4.1934 idF der Bek. v. 1.4.1960, GBl. 1960, S. 37; Landesverwaltungsgesetz des Landes BadenWürttemberg v. 7.11.1955, GBl. 1955, Nr. 22 S. 225; Erstes Vereinfachungsgesetz des Landes Nordrhein-Westfalen v. 23.7.1957, GV. 1957, Nr. 50 S. 189; Gesetz über das Verfahren der Berliner Verwaltung v. 2.10.1958, GVBl. 1958, S. 951; Allgemeines Verwaltungsgesetz für das Land Schleswig-Holstein v. 18.4.1967, GVOBl. 1967, S. 131; vgl. auch BT-Drucks. 7/910, S. 31.

9 Vgl. Entwurf eines Gesetzes zur Regelung des Verwaltungsverfahrens im Lande Niedersachsen, LT-Drucks. Nr. 1572 v. 28.09.1954; Referentenentwurf eines Verwaltungsverfahrensgesetzes für das Land Nordrhein-Westfalen (Stand 1960) und Referentenentwurf eines Hamburger Verwaltungsgesetzes (Stand 1960), vgl. auch BT-Drucks. 7/910, S. 31.

10 Vgl. Musterentwurf eines Verwaltungsverfahrensgesetzes (EVwVerfG 1963) mit Anhang „Münchener Fassung“, 2. Aufl. 1968.

11 BT-Drucks. VI/1173, Schübel-Pfister, in: Mann/Sennekamp/Uechtritz (Hrsg.), VwVfG, 2. Aufl. 2019, \$23 Rn. 1.

$12 v$. Ebner, DVBl. 1971, 341.

13 v. Ebner, DVBl. 1971, 341 (341). 
Es liegt nahe, dass es weniger die Handelsreisenden gewesen sein dürften, die die Verwaltung vor Sprachhindernisse stellten als die „Gastarbeiter:innen" 14 , also jene Ausländer:innen, die in Deutschland lebten und leben. Von Ebner verweist in der Frage, wie nun mit ausländischen Personen umzugehen sei, zunächst darauf, dass es dem Verwaltungsverfahrensrecht an einer sprachregelnden Vorschrift vergleichbar zu $₫ 184$ GVG fehle. ${ }^{15}$ In Ermangelung einer solchen Regelung erforderten das Diskriminierungsverbot aus Art. 3 Abs. 3 GG („wegen seiner Sprache“), das Sozialstaatsprinzip aus Art. 20 Abs. 1 GG und der Grundsatz des rechtlichen Gehörs in Verbindung mit den Grundsätzen der Nichtförmlichkeit und der Ermittlung von Amts wegen „eine weitgehende Gleichbehandlung von In- und Ausländern im Verwaltungsverfahren“. ${ }^{16}$

Diese Analyse findet sich im unmittelbar darauffolgenden Vorschlag allerdings nicht wieder. Von Ebner schlägt vor, dass, sofern auf Seiten der Behörde keine Sprachkenntnisse vorliegen, die Behörde von dem oder der Verfahrensbeteiligten eine Übersetzung verlangen oder eine Übersetzung selbst veranlassen kann, deren Kosten von der ausländischen Person zu tragen sind. ${ }^{17}$ „Weitgehende Gleichbehandlung“ bedeutet also nicht etwa, dass ausländische Personen, ebenso wie Deutsche, sich in der ihnen verständlichen Sprache an die öffentliche Verwaltung wenden können, sondern, dass für deutschunkundige Ausländer:innen sowie für deutschsprachige Inländer:innen, trotz ihrer Ungleichheit, die Verwaltungssprache Deutsch zu seien habe. Die Verantwortung und damit auch das Sprachrisiko $^{18}$, also das Risiko des Nicht- oder Fehlverstehens, will von Ebner allein auf der Seite der ausländischen Personen sehen.

Dieser erste Aufschlag aus Verwaltungsperspektive wird wenige Jahre später die gesetzliche Regelung der Amtssprache prägen. Nicht nur werden Gesetz und Gesetzesbegründung in Aufbau und Ergebnis unüberseh-

14 Aufgrund des aus mehrfachen Perspektiven problematischen Begriffs des „Gastarbeiters“, der allerdings als zeitgeschichtlicher Begriff unentbehrlich ist, werden Anführungszeichen verwendet.

15 v. Ebner, DVBl. 1971, 341 (344).

16 v. Ebner, DVBl. 1971, 341 (344).

17 v. Ebner, DVBl. 1971, 341 (344), vgl. dort Fn. 23.

18 Die Übertragung dieses zivilrechtlichen Begriffs auf das Verwaltungsverfahren geht zurück auf Ingerl, Sprachrisiko im Verfahren, 1988, insbes. S. 6. ff. 
bar eng an von Ebners Ausführungen und Vorschlägen liegen, ${ }^{19}$ die Begründung wird auch explizit auf seinen Beitrag verweisen. ${ }^{20}$

\section{Der Regierungsentwurf von 1973: Lex „Gastarbeiter“}

Noch unter $₫ 19$ Abs. 1 enthält erstmals der Regierungsentwurf von 1973 eine Regelung zur Amtssprache. ${ }^{21}$ Der Wortlaut ist damals so schlicht wie heute: Die Amtssprache ist deutsch. Aufschlussreicher ist hingegen die Begründung. Scheint zunächst unklar, wie ein Rechtsinstitut, das in allen vorherigen Entwürfen und Vorläufern nicht vorkommt, sich den Weg in den zweiten Regierungsentwurf bahnt, so gibt die Begründung Auskunft:

„Während $\$ 184$ des Gerichtsverfassungsgesetzes für das gerichtliche Verfahren die deutsche Sprache als Gerichtssprache für verbindlich erklärt, fehlt für das Verwaltungsverfahren bisher eine entsprechende Vorschrift. Das Fehlen einer solchen Vorschrift gewinnt indessen in der Praxis zunehmende Bedeutung (...).

In der Bundesrepublik Deutschland sind augenblicklich etwa 2,4 Millionen ausländische Arbeitnehmer beschäftigt, darunter ca. 528000 Türken, 466000 Jugoslawen, 410000 Italiener, 268000 Griechen, 180000 Spanier, 69000 Portugiesen, 15300 Marokkaner und 11200 Tunesier (...). Schon die Vielfalt der Herkunft der Arbeitnehmer zeigt das Sprachenproblem auf, vor das sich die Verwaltung gestellt sieht. “22

An dieser Begründung ist zunächst bemerkenswert, dass sie offen eine bestimmte Gruppe benennt, die in den Augen des Gesetzgebers eine Amtssprachenregelung notwendig machte: $\mathbb{1 9}$, der spätere $\$ 23 \mathrm{VwVfG}$, ist ein Gesetz für und über „Gastarbeiter:innen“. Ebenso kann verwundern, dass auf die Sprachbarrieren zwischen deutschunkundigen Verfahrensbeteiligten und Verwaltungsbehörden nicht problemadäquat und lösungsorien-

19 Aufbau und Begründung sind fast identisch, beide beginnen mit dem Verweis auf das Fehlen einer zu $₫ 184$ GVG vergleichbaren Norm im VwVfG, nennen dann Ausländerzahlen; die Formulierung des $₫ 23$ VwVfG folgt zudem den Vorschlägen von Ebners.

20 BT-Drucks. 7/910, S. 48.

21 P. Stelkens/Schmitz, in: Stelkens/Bonk/Sachs VwVfG (Fn. 4), $\$ 23$, Entstehungsgeschichte; BT-Drucks. 7/910, S. 12.

22 BT-Drucks. 7/910, S. 48. 
tiert reagiert wurde, sondern die Problematik lediglich auf die Seite der "Gastarbeiter:innen“ verlagert wurde. ${ }^{23}$

Soweit heute argumentiert wird, $\$ 23$ VwVfG stelle nicht auf den Personenkreis ab, der die Erklärung abgibt, sondern auf die in der Erklärung benutzte Sprache, ${ }^{24}$ so widerspricht dies nicht nur der Gesetzesbegründung, rechtstatsächlich kommt hinzu, dass die Zahl der deutschunkundigen deutschen Staatsbürger wohl verschwindend gering sein dürfte.

\section{Rechtspolitische Stoßrichtung der Amtssprachenregelung}

Dass die Gesetzesbegründung die „Gastarbeiter:innen“ in ein problematisierendes Narrativ einbettet („Schon die Vielfalt der Herkunft der Arbeitnehmer zeigt das Sprachenproblem auf, vor das sich die Verwaltung gestellt sieht.“" ${ }^{25}$ ), zeigt, wie sehr die Genese des $₫ 23$ VwVfG von Zeitgeist und Debatten der 1970er Jahre geprägt war. Wurden „Gastarbeiter:innen“ zuvor noch als Garanten des Wirtschaftswachstums angesehen, so erklärte man sie 1966/67 zum Sündenbock der eintretenden Rezession. ${ }^{26}$ Es begann eine politische Trendwende, die über breite Diskussionen um Familiennachzug und gestiegene Arbeitslosenzahlen am 23. November 1973, im selben Jahr des Regierungsentwurfs zum VwVfG, im sogenannten Anwerbestopp für ausländische Arbeitskräfte ihren Höhepunkt fand. ${ }^{27}$

Auch wenn die Rechtspolitik der frühen 1970er Jahre ausländische Arbeitnehmer als ein vorübergehendes (Stör-)Phänomen wahrnahm, waren sie längst Teil der gesellschaftlichen Realität. Nach dem Anwerbestopp stieg ihre Zahl sogar von 2,4 Millionen $1973^{28}$ auf 4,4 Millionen im Jahre 1980 an. ${ }^{29}$ Dem entgegen hat Deutsch als Amtssprache die Sprachproble-

23 Ähnlich Götz, NJW 1976, 1425 (1427), Häberle, Verfassungsprinzipien „im“ Verwaltungsverfahrensgesetz, in: Schmitt Glaeser (Hrsg.) Verwaltungsverfahren. Festschrift zum 50-jährigen Bestehen des Richard Boorberg Verlags, 1977, S. 47 (62).

24 Schmitz, in: Stelkens/Bonk/Sachs VwVfG (Fn. 4), \$23 Rn. 3; Kugele, VwVfG, 2014, $\$ 23$ Rn. 3: „unabhängig von wem sie kommen“.

25 BT-Drucks. 7/910, S. 48.

26 Siehe hierzu instruktiv Herbert, Geschichte der Ausländerpolitik, 2. Aufl. 2017, S. 218 ff. und Meier-Braun, Deutschland, Einwanderungsland, 2002, S. 36.

27 Meier-Braun, Einwanderungsland (Fn. 26), S. 42.

28 BT-Drucks. 7/910, S. 48.

29 Meier-Braun, Einwanderungsland (Fn. 26), S. 42; Grund hierfür war auch der Anwerbestopp: durch die versperrte Möglichkeit einer Rückkehr blieben viele der „Gastarbeiter:innen“ in Deutschland. 
me nicht aufgehoben, sondern lediglich aus dem Verantwortungsbereich der Behörden entfernt.

\section{Inkrafttreten und Ergänzungen der deutschen Amtssprache}

Am 15. Januar 1976 hat das Plenum des Bundestages den Gesetzentwurf von 1973 mit unwesentlichen Änderungen ${ }^{30}$ verabschiedet, ${ }^{31}$ am 1. Januar 1977 trat das Verwaltungsverfahrensgesetz des Bundes in Kraft. ${ }^{32}$ Dort findet sich die Amtssprachenregelung aus $\$ 19$ Abs. 1 des Regierungsentwurfs von 1973 mit gleichem Wortlaut in $\$ 23$ Abs. 1 VwVfG.

Jene Verwaltungsverfahrensgesetze der Länder, die nicht ohnehin auf das BVwVfG verweisen, haben den Grundsatz der deutschen Amtssprache wortgleich übernommen.

Seit Inkrafttreten der Europäischen Charta der Regional- oder Minderheitensprachen am 1. Januar 1999 wird Deutsch als Amtssprache in bestimmten Regionen durch ihr gleichgestellte Minderheitensprachen ergänzt. ${ }^{33}$ Als Minderheitensprachen geschützt sind Dänisch, Ober- und Niedersorbisch, Nord- und Saterfriesisch und das Romanes der deutschen Sinti und Roma; Niederdeutsch ist geschützte Regionalsprache. ${ }^{34}$ In Umsetzung des Art. 10 der Charta sieht zum Beispiel $\$ 1$ Abs. 2 S. 1 Friesisch-Gesetz $^{35}$ vor, dass vor Behörden im Kreis Nordfriesland und auf der Insel Helgoland auch die friesische Sprache verwendet werden darf. Diese Ausnahmen vom Grundsatz der deutschen Amtssprache dürften allerdings primär dem Kulturschutz der Minderheitensprachen dienen ${ }^{36}$ als unmittelbar auf

30 Änderungsvorschläge des Bundesrats die Amtssprachenregelung betreffend, waren nur auf die Absätze 2 und 4 bezogen, insbesondere sollte Abs. 2 klarstellen, dass die Kosten der Übersetzung von den Verfahrensbeteiligten zu tragen seien, hierzu Eichler, VwVfG, Bd. I, Stand: 1.9.1985, $\mathbb{} 23$.

31 Ule, Die Kodifizierung des Verwaltungsverfahrensrechts, in: Jeserich/Pohl/von Unruh (Hrsg.), Deutsche Verwaltungsgeschichte, Bd. V, 1987, S. 1174; Deutscher Bundestag, Plenarprotokoll v. 15.01.1976, S. 14695.

32 \103 Abs. 1 VwVfG a.F., vgl. BGBl. 1976 I, S. 1277.

33 Gesetz zu der Europäischen Charta der Regional- und Minderheitensprachen des Europarats vom 6. November 1992, BGBl. 1998 II, S. 1314.

34 Europäische Charta der Regional- und Minderheitensprachen (Fn. 33), S. 1334.

35 Gesetz zur Förderung des Friesischen im öffentlichen Raum, GVOBl. 2004, S. 481.

36 Dass dies die vorrangige Bestimmung der Charta ist, wird schon aus Art. 7 deutlich, der die Ziele und Grundsätze der Charta aufzählt. In Teil III folgen sodann die Maßnahmen zur Umsetzung dieser Ziele, als eine Maßnahme unter vielen fin- 
Rechtsschutz der Verfahrensbeteiligten gerichtet zu sein, sprechen wohl diejenigen, die Minderheitensprachen beherrschen, auch Deutsch. ${ }^{37}$ Auch spezielle Verwaltungsverfahren machten Ergänzungen notwendig. So offenbart sich die angedeutete Problematik um eine Verlagerung des sog. Sprachrisikos in die Sphäre der deutschunkundigen Personen insbesondere anhand notwendiger Sonderregelungen für spezielle Verwaltungsverfahren wie das Asylverfahren. ${ }^{38}$

\section{Die historische Entwicklung der deutschen Amtssprache}

Ausgehend von den politischen Umständen der derzeitigen Rechtslage, lohnt ein Blick in die historische Entwicklung der deutschen Amtssprache. Vorauszustellen ist, dass im Rückblick vielfach nicht von einer Amtssprachenregelung im heutigen Sinne gesprochen werden kann. ${ }^{39}$ Verwaltung und Verwaltungsrecht im heutigen Sinne, auf deren Vorliegen auch unsere Vorstellungen einer Amtssprache basieren, entwickelten sich erst im Ausgangspunkt des 17. und 18. Jahrhunderts und formten sich schließlich in einer national-bürgerlichen Epoche aus. ${ }^{40}$ Nötig ist es daher, auch die Entwicklung der Rechts- und Gesetzessprache in die Betrachtungen miteinzubeziehen.

det sich auch, dass der Gebrauch von Minderheitensprachen vor den Verwaltungsbehörden zu ermöglichen ist.

37 Der Erste Bericht der Bundesrepublik Deutschland gem. Artikel 25 Absatz 1 des Rahmenübereinkommens des Europarates zum Schutz Nationaler Minderheiten hält hierzu fest: „Die Angehörigen der Minderheit verstehen Dänisch und sprechen diese Sprache zum größten Teil. Alle beherrschen zudem Deutsch." (S. 5); „Etwa 35000 Sorben beherrschen noch die sorbische Sprache in Wort und Schrift; alle Sorben sprechen auch Deutsch.“ (S.5); für die Friesen fehlt es an einer expliziten Angabe, allerdings verstehen die Friesen sich auch nicht als Minderheit, sondern eher als Gruppe mit eigener Sprache innerhalb Deutschlands, was nahelegt, dass auch sie vollständig Deutsch beherrschen (S. 8), abrufbar unter https:/www.bmi.bund.de/SharedDocs/downloads/DE/veroeffentlichungen/them en/heimat-integration/minderheiten/1-erster-staatenbereicht-rahmenuebereinkom men.pdf?_blob=publicationFile\&v=3 (letzter Aufruf am 29.04.2021).

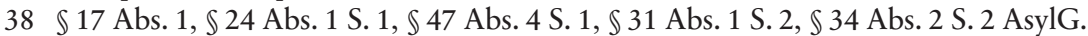

39 Ähnlich Bader, Recht - Geschichte - Sprache, in: Historisches Jahrbuch 93 (1973), 1 (14): „Sonderformen der Rechtssprache, wie etwa die Verwaltungssprache, die Sprache der Behörden, (...), gehören Epochen einer ausgebildeten Staatsorganisation (...) an."

40 Feist, Die Entstehung des Verwaltungsrechts als Rechtsdisziplin, 1968, S. 1. 
Dennoch gab es Kommunikation zwischen Souverän und Untertanen, zwischen Herrscher und Beherrschten, was sich auch am Heiligen Römischen Reich zeigen lässt.

\section{Heiliges Römisches Reich}

Für das Heilige Römische Reich können wir schon deshalb nicht von Amtssprache nach heutigem Verständnis sprechen, weil der Begriff der Verwaltung nicht streng juristisch-bürokratisch verstanden werden kann, eher fungiert er für das Heilige Römische Reich als eine Umschreibung für die Art und Weise der Verwirklichung des Willens der zentralen Gewalt. ${ }^{41}$ Diese Verwaltung war eng an Hof und Territorium gebunden, sodass die landesherrliche Gewalt gewissermaßen verdinglicht war, die Ausgangsbedingungen für einen Blick auf die Amtssprache sind also völlig andere als gegenwärtig. ${ }^{42}$

\section{a) Sprachliche Kurfürstenausbildung in der Goldenen Bulle}

Dennoch stammt der älteste, die Sprache betreffende Rechtstext aus der Goldenen Bulle ${ }^{43}$. Der Gesetzestext, der 1356 von Karl IV. als Urkunde verkündet wurde, regelte die Verfahren für Wahl und Krönung des römisch-deutschen Königs sowie die Rechte und Pflichten der Kurfürsten.

In ihrem 31. Titel finden sich Regelungen über die Ausbildung, der Kurfürstensöhne, den zukünftigen Regenten der Territorien:

„Da das Heilige Römische Reich in seiner Würde die Gesetze und Regenten der verschiedenen Länder lenkt, die sich durch Gebräuche, Lebensweise und Sprachen unterscheiden, ziemt es sich und wird nach der Meinung aller weisen Leute für nützlich befunden, wenn man die Kurfürsten - die Pfeiler und Mauern des Reiches - über die Verschiedenheiten der Mundarten und Sprachen belehrt. Sie sollen viele Leute

41 Moraw, Die Verwaltung des Königtums und des Reiches und ihre Rahmenbedingungen, in: Jeserich/Pohl/von Unruh (Hrsg.), Deutsche Verwaltungsgeschichte, Bd. I, 1983, 1 S. 22.

42 Feist, Rechtsdisziplin (Fn. 46), S. 3; ähnlich Moraw, Verwaltung (Fn. 40), S. 32, 50.

43 Hattenhauer, Zur Geschichte der deutschen Rechts- und Gesetzessprache, in: Berichte aus den Sitzungen der Joachim-Jungius-Gesellschaft der Wissenschaften e.V., 1987, Jg. 5, Heft 2, S. 6. 
verstehen und von vielen verstanden werden, um den erhabenen Kaiser bei der Sorge für die Nöte so vieler Menschen zu unterstützen." ${ }^{44}$

$\mathrm{Zu}$ den Sprachen, die Kurfürstensöhne zu beherrschen hatten, zählten neben dem Deutschen, das Lateinische, das Italienische und das Slawische. Auch wenn eine Art von Kommunikation zwischen (zukünftigem) Souverän und Untertanen bestanden haben muss („sie sollen viele Leute verstehen") und eine diesbezügliche Sprachenregelung notwendig war, werden mit dieser Passage nicht etwa en passant alle vier aufgezählten Sprachen zu Amtssprachen deklariert. Die verordnete Sprachenkenntnis der Kurfürsten sollte vielmehr sicherstellen, dass die Kurfürsten der Sprachenvielfalt des Reiches auch gewachsen waren. ${ }^{45}$ Die zitierte Passage lässt sich also als Bekenntnis zur sprachlichen Vielfalt des Reiches verstehen, ${ }^{46}$ die in ihrer Substanz nicht anzutasten war, ${ }^{47}$ sondern auf die die Kurfürsten angemessen zu reagieren hatten.

\section{b) Reichssprachen}

Neben den Regelungen zur Kommunikation zwischen Kurfürsten und Volk gab es auch offizielle Reichssprachen, also „Staatssprachen“ im Heiligen Römischen Reich: 1519 wurden mit der ersten Wahlkapitulation - eine Selbstverpflichtung des Kaisers gegenüber dem wählenden Kurfürstenkollegium - Deutsch und Latein zu Reichssprachen erklärt. ${ }^{48}$

Art. XIV der Wahlkapitulation Karls V. vom 3. Juli 1519 legt zwar Deutsch und Latein für Schriften und Handlungen des Reichs fest, sieht aber auch die Berücksichtigung anderer ortsüblicher Sprachen vor („es were dann an Orten, da gemeinlich ain anndere Sprach in Ubung und Gebrauch stuende, alsdann mögen Wir und die unnsern Unns derselben daselbs auch behelffen"). ${ }^{49}$ Auch diese Norm kann in Anknüpfung an die

44 Mit Hervorheb. d. Verfasserin zitiert nach Fritz/Müller-Mertens, Die Goldene Bulle - Das Reichsgesetz Kaiser Karls IV. vom Jahre 1356, übersetzt v. Wolfgang D. Fritz, 1978, S. 88.

45 Schneider, Sprachenpolitik im Mittelalter, in: Marti (Hrsg.), Sprachenpolitik in Grenzregionen, 1996, S. 65 (75).

46 So auch Hattenhauer, Geschichte (Fn. 43), S. 7.

47 Schneider, Sprachenpolitik (Fn. 45), S. 75.

48 von Polenz, Deutsche Sprachgeschichte vom Spätmittelalter bis zur Gegenwart, Bd. II, 2. Aufl. 2013, S. 55.

49 Burgdorf (Bearb.), Die Wahlkapitulationen der römisch-deutschen Könige und Kaiser 1519-1792, 2015, S. 26. 
sprachliche Kurfürstenausbildung als ein Anerkenntnis des sprachlichen Pluralismus des Reiches gelesen werden. Zwar werden Deutsch und Latein Vorrangstellungen eingeräumt, gleichzeitig schien es aber rechtspraktisch notwendig, hiervon ortsbedingt abzuweichen.

Dieses multilinguale Selbstverständnis des Reiches, formuliert durch den Kaiser, wird sich, an verschiedenen Stellen und mit abgewandeltem Wortlaut, aber im Regelungskern gleich, bis zur Wahlkapitulation Lepolds I. vom 18. Juli 1658 wiederholen. ${ }^{50}$ Ab 1658 allerdings wurde die Verwendung anderer Sprachen auf Gebiete außerhalb des Reiches beschränkt. ${ }^{51}$ Eine mögliche Erklärung für diese Einschränkungen könnten jedenfalls für 1682 und 1717 nachgewiesene Konflikte mit französisch-diplomatischen Gesandten auf den Reichstagen gewesen sein. ${ }^{52}$

Insgesamt war die Sprachenpolitik des Heiligen Römischen Reiches durch bemerkenswerte Bemühungen der Sprachenvielfalt des Großreiches gerecht zu werden, gekennzeichnet. Begegnet wurde der natürlich gewachsenen Multilingualität des Reiches nicht etwa aggressiv oder betont kämpferisch, sondern vielmehr bewusst entsprechend. ${ }^{53}$

\section{Rezeption und Einfluss des Lateinischen}

Teils parallel zu den oben geschilderten Entwicklungen vollzog sich die Rezeption des römischen Rechts. Bereits im 11. Jahrhundert nimmt in Italien die Wiederbelebung der römischen Rechtskultur ihren Anfang. In Deutschland begann die praktische Rezeption des römischen Rechts im Spätmittelalter mit dem Studium deutscher Scholaren an oberitalienischen Universitäten. Dort in römischem Recht ausgebildet, trugen die Juristen das neue Recht und seine Sprache in die Kanzleien und Höfe hinein. ${ }^{54}$

Beides, die Rezeption des römischen Rechts als auch die Übernahme der Sprache des römischen Rechts, Latein, waren fließende Prozesse, die

50 Vgl. Burgdorf, Wahlkapitulationen (Fn. 49), S. 38, 52, 66, 82, 97, 116, 137, 180.

51 Burgdorf, Wahlkapitulationen (Fn. 49), S. 226.

52 Auch schon vorher soll es Ordnungsstrafen des Reichskammergerichts gegen unbotmäßige Prokuratoren gegeben haben, was den Kaiser zur Einschränkung der doch sehr weiten Formulierung bewogen haben könnte, vgl. von Polenz, Sprachgeschichte Bd. II (Fn. 48), S. 55 f.; ähnlich: Hattenhauer, Geschichte (Fn. 43), S.9, $11 \mathrm{f}$; mit Verweis auf Moser, Abhandlungen von der Teutschen Sprache, in: Vermischte Schriften über mancherley das Teutsche Staats=Recht betreffende Materien, 1733, S. 414.

53 Schneider, Sprachenpolitik (Fn. 45), S. 77.

54 Bartsch, AcP 1954, 412 (417). 
nie ganz vollendet wurden. Auch weil viele Gesetzestexte weiterhin in deutscher Sprache abgefasst waren, vollzog sich nicht jedwede Rechtskommunikation in lateinischer Sprache. So bestimmte beispielsweise eine Verordnung für die Kanzlei des Herzogs von Zweibrücken von 1586: „Die Sekretäre sollen die Geschäfte halten in guter, geschickter, lauterer und unverdunkelter kanzleiischer Form mit guten deutschen und nit anderen Wörtern". 55

Dennoch kann davon ausgegangen werden, dass die „Überfremdung“ der deutschen Rechtssprache bis ins 18. Jahrhundert stetig zunahm. ${ }^{56}$ Jedenfalls war die Rechtssprache seit der Rezeption mit lateinischen Begriffen durchzogen und für Laien nur noch schwer verständlich. In der Spätphase der Rezeption des römischen Rechts zeigt sich damit auch das Herrschaftspotenzial von Sprache: während subalterne Hofbedienstete dem Deutschen zugeneigt waren, beharrten akademische Juristen aus ihren höheren Ämtern heraus auf dem ihnen vertrauten Latein als Standessymbol. ${ }^{57}$

Kritisch zusammengefasst versperrte dieses ,Juristenkauderwelsch (...) den lateinunkundigen Volksgenossen wie mit einem stacheligen Draht-

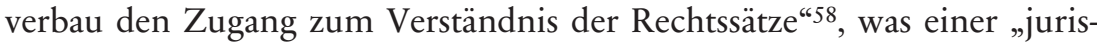
diktionell[en] Entmündigung der nicht-akademischen und nichtfeudalen Stände" gleichkam. 59

\section{Das 17. und 18. Jahrhundert}

Auch wenn das Festhalten am Latein bis Mitte des 18. Jahrhunderts stark ausgeprägt war, ${ }^{60} \mathrm{kam}$ in dieser Zeit Kritik an der lateinisch geprägten Rechtssprache auf.

Mit Ende des Dreißigjährigen Krieges 1648 und angetrieben von den Erschütterungen der vielen Kriegsjahre, machte es sich eine aufkommende

55 Zitiert nach Merk, Werdegang und Wandlungen der deutschen Rechtssprache, 1933, S. 24.

56 Merk, Werdegang (Fn. 55), S. 24.

57 von Polenz, Sprachgeschichte Bd. II (Fn. 48), S. 56.

58 Merk, Werdegang (Fn. 55), S. 23.

59 von Polenz, Deutsche Sprachgeschichte - Vom Spätmittelalter bis zur Gegenwart, Bd. I, 1991, S. 216; Görgen, Aufklärerische Tendenzen in der Gesetzessprache der frühen Neuzeit, in: Kronauer/Garber (Hrsg.), Recht und Sprache in der deutschen Aufklärung, 2001, S. 81.

60 von Polenz, Sprachgeschichte Bd. II (Fn. 48), S. 56, 409. 
Bewegung zur Aufgabe die deutsche Sprache zu schützen. Die sog. Sprachgesellschaften fürchteten eine kulturelle und sprachliche Überfremdung des Reichs und versuchten seine innere Zerrissenheit über die Hoheit und Reinhaltung der deutschen Sprache abzuwehren. ${ }^{61}$ Auch wenn diese Vereinigungen keinen Einfluss auf die Rechtssprache hatten, ${ }^{62}$ trat mit den selbsternannten Sprachreinigern doch eine Wendung ein, die bis heute fortwirkt. Als Erste politisierten sie Sprache und idealisierten eine Einheit von Nation, Volk und Sprache.

\section{a) Aufklärung und Rechtssprache}

Es war schließlich die Aufklärung und ihre Ideen, die die Abwendung vom Lateinischen brachten. Von Hermann Conring über Montesquieu bis Cesare Beccaria forderten alle eine Rechts- und Gesetzessprache, die für Bürger verständlich sein sollte. ${ }^{63}$ Auch Gottfried Wilhelm Leibniz verlangte bereits 1667 eine Übersetzung des Corpus iuris ins Deutsche und Disputationen auf Deutsch. ${ }^{64}$

In diesem Sinne reformierten einige Rechtsgelehrte das rechtswissenschaftliche Studium: Christian Thomasius und Christian Wolff hielten erstmals Vorlesungen in deutscher Sprache und trieben so die Wortfindung für eine von lateinischen Begriffen unabhängige deutsche Rechtssprache an. ${ }^{65}$ Dennoch sollte die aufklärerische Reform der deutschen Rechtssprache der vorangegangenen Entwicklung der wissenschaftlichen Rechtssprache um fast ein Jahrhundert verzögert nachfolgen. ${ }^{66}$

\section{b) Die Auswirkungen auf die Amtssprache}

Als Leibniz die Gründung der Berliner Akademie der Wissenschaften betreute, erteilte der damalige Kurfürst Friedrich III. von Brandenburg und

61 Hattenhauer, Geschichte (Fn. 43), S. 15.

62 Hattenhauer, Geschichte (Fn. 43), S. 19 ff.

63 Hattenhauer, Geschichte (Fn. 43), Conring forderte dies ironischerweise auf Latein, S. 36, 38 u. 40.

64 Hattenhauer, Geschichte (Fn. 43), S. 24, mit Verweis auf Hartmann (Hrsg.), Leibniz als Jurist und Rechtsphilosoph, Neudruck 1974, $\$ 65$.

65 Hattenhauer, Geschichte (Fn. 43), S. 28.

66 von Polenz, Sprachgeschichte Bd. II (Fn. 48), S. 409. 
Herzog in Preußen ihm 1700 den Auftrag, auch das Werden einer neuen, einer deutschen Kanzleisprache zu unterstützen:

„(...) daß in Unsern Kantzleyen, Regierungen, Collegien und Gerichten bey denen Ausfertigungen die frembde unanständige Worte und übel entlehnte Reden, so viel füglich geschehen kann, vermieden, hingegen gute teutsche Redearten erhalten, herfürgesuchet und vermehret werden, also wollen Wir auch Verordnung machen, daß der Sozietät mit teutschen Benennung- und Beschreibungen an Hand gegangen $(\ldots)^{* 67}$

Nicht nur wurde damit die Amtshilfe der Behörden zugesagt, es wurde auch der Auftrag erteilt aus deren Amtssprache, einer latinisierten elitären Standessprache, ${ }^{68}$ eine für alle gleichermaßen verständliche deutsche Amtssprache werden zu lassen.

Zwar obskur, aber dafür von parabolischem Wert ist die Erzählung von Maria Theresia und dem buta ember. ${ }^{69}$ Die Reformerin Maria Theresia soll angeordnet haben, ${ }^{70}$ dass jeder Entwurf einer behördlichen Anordnung einem Mann mit Grundschuldbildung und durchschnittlicher Intelligenz, einem buta ember (wörtlich übersetzt: einem dummen Menschen) vorgelegt werden sollte. ${ }^{71}$ Konnte der buta ember den Inhalt der Anordnung nicht frei wiedergeben, so musste der Entwurf umgeschrieben werden. Damit legte Maria Theresia größten Wert auf eine allgemeine Verständlichkeit der Anordnungen.

Ein weiterer Vorstoß geht auf Joseph II., den Sohn Maria Theresias, zurück, der die reformatorische Politik seiner Mutter fortsetzte. Nach dem Sprachedikt vom 20. Februar $1784^{72}$ sollte im gesamten Herrschaftsgebiet Deutsch an die Stelle des Lateins als Amtssprache treten.

67 Zitiert nach Hattenhauer, Geschichte (Fn. 43), S. 26, mit Hinweis auf Schmied-Kowarzik (Hrsg), G.W. Leibniz, Deutsche Schriften, Erster Band: Muttersprache und völkische Gesinnung, 1916, S. 71.

68 Hattenhauer, Geschichte (Fn. 43), S. 26.

69 Schönherr, Sprache und Recht - Aufsätze und Vorträge, hrsgg. v. Barfuß, 1985, S. 83.

70 Es handelt sich hierbei um eine mündlich weitergetragene Erzählung für die es keinen aktenmäßigen Beleg gibt, Schönherr, Sprache und Recht (Fn.69), S. 83; Pfeiffer/Stroubal/Wodak, Recht auf Sprache - Verstehen und Verständlichkeit von Gesetzen, 1987, S. 5 Fn. 6.

71 Pfeiffer/Stroubal/Wodak, Recht auf Sprache (Fn. 70), S. 5.

72 Mit Nachweis bei Hattenhauer, Lingua vernacula - Rechtssprache zwischen Volkssprache und Gelehrtensprache, in: Eckert/Hattenhauer (Hrsg.), Sprache - Recht Geschichte, 1991, S. 49 (68). 
Schon in dem 1785 veröffentlichten Werk „Über den Geschäftsstil“, einem Lehrbuch für angehende Beamte, bemerkt Joseph von Sonnenfels folgendes über die Gesetzessprache und ihre Bedeutung für den Geschäftsstil, also die Sprache der Behörden:

„Das Volkswort, das Provinzialwort (...) das in jedermanns Munde ist, muss hier der Sprache der Wielande und Lessinge vorgezogen werden. (...) Alles, was man sich beim Verfassen der Gesetze erlaubt, ist, daß dem unrichtigen aber gangbaren Ausdrucke der richtige eingeklammert an die Seite gestellt werde. “73

Dieser Einblick zeigt, dass die Gesetze nun in deutscher Sprache verfasst waren, höchstens wurden noch erklärend lateinische Begriffe in Klammern beigefügt. Als 1794 das Allgemeine Landrecht für die Preußischen Staaten eingeführt wird, entsteht so auch ein Gesetzeshochdeutsch für das gesamte Reich. ${ }^{74}$ Gegen Ende des 18. Jahrhunderts war Latein als Rechtssprache also endgültig abgelöst. Durch die Primatstellung der Gesetzessprache, auf die sich die Amtssprache noch heute notwendigerweise zu beziehen hat, bestimmte die deutsche Rechtssprache nun auch eine deutsche Amtssprache, die der Idee einer egalitären Verständlichkeit gerecht werden konnte.

\section{Minderheitenrechte in der Paulskirchenverfassung}

1848/1849 kam auch in der Paulskirche die Verständlichkeit der Rechtssprache auf, nun aber erstmals aus der Perspektive der Rechte sprachlicher Minderheiten. Diese Minderheitenrechte bauten ideengeschichtlich auf dem aufklärerischen Postulat der Verständlichkeit auf und führten es fort. In $₫ 188$ der Reichsverfassung vom 27. März 1849 heißt es:

„Den nicht deutsch redenden Volksstämmen Deutschlands ist ihre volksthümliche Entwickelung gewährleistet, namentlich die Gleichberechtigung ihrer Sprachen, so weit deren Gebiete reichen, in dem Kirchenwesen, dem Unterrichte, der innern Verwaltung und der Rechtspflege. " 75

73 von Sonnenfels, Über den Geschäftsstil - Die ersten Grundlinien für angehende österreichische Kanzleibeamten, 2. Aufl. 1785, zitiert nach Hattenhauer, Geschichte (Fn. 43), S. $37 \mathrm{f}$.

74 Hattenhauer, Geschichte (Fn. 43), S. 58.

75 Hervorheb. d. Verfasserin. 
Auch wenn die Berücksichtigung von Minderheitenrechten an Gebiete geknüpft war, kann man diese Regelung als progressiv beschreiben. ${ }^{76} \$ 188$ der Paulskirchenverfassung lässt sich das gesetzgeberische Ziel entnehmen, durch Sprachenregelungen den Zugang zu Recht für möglichst viele Menschen zu ermöglichen und zu gewährleisten. Insoweit handelt es sich um eine erweiterte Fortführung der aufklärerischen Idee von der Verständlichkeit der Kommunikation zwischen Staat und Bürger, die nun noch inklusiver auch auf nicht deutsch redenden Volksstämme Deutschlands bezogen wurde.

\section{Die Wende in der Sprachenpolitik}

Diese sprachenpolitische Ausrichtung wird sich mit dem aufkommenden Nationalismus grundlegend ändern. Dieser postulierte eine Einheit von Nation, Volk und Sprache und ließ Minderheitensprachen keinen Raum mehr.

\section{Das Geschäftssprachegesetz von 1876}

Aktuelle Gesetzeskommentare zu $₫ 23$ VwVfG verweisen auf ein Vorgängergesetz aus Preußen, ${ }^{77}$ mit dem die Amtssprachenregelung des VwVfG in eine gesetzgeberische Tradition eingegliedert werden soll:

„Immerhin existierte in Preußen bereits seit dem Jahre 1876 das Gesetz vom 28. August 1876, betreffend die Geschäftssprache der Behörden, Beamten und politischen Körperschaften. Dessen $₫ 1$ schrieb vor, dass die deutsche Sprache die ausschließliche Geschäftssprache war und auch der schriftliche Verkehr mit den angesprochenen Stellen in deutscher Sprache stattzufinden hatte. ${ }^{\text {778 }}$

76 Häberle, Sprachen-Artikel und Sprachenprobleme in westlichen Verfassungsstaaten, in: Brem/Druey/Kramer/Schwander (Hrsg.), Festschrift zum 65. Geburtstag von Mario M. Pedrazzini, 1990, 105 (111) spricht von einem „Leitartikel zum Schutz sprachlicher Minderheiten".

77 Heßhaus, in: Bader/Ronellenfitsch (Hrsg.), BeckOK VwVfG, 50. Ed. Stand: 01.01.2021, \$23 Rn.1.1; Ritgen, in: Knack/Henneke (Hrsg.), VwVfG, 11. Aufl.

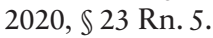

78 Hervorheb. d. Verfasserin, Heßhaus, in: BeckOK (Fn. 77), $\$ 23$ Rn. 1.1. 
Auch wenn eine preußische Rechtstradition des $₫ 23 \mathrm{VwVfG}$ nahezuliegen scheint, entsteht so ein missverständliches Bild, lässt diese rechtsgeschichtliche Einordnung doch die Motive des Geschäftssprachegesetzes völlig unbeachtet.

\section{a) Politische und gesellschaftliche Umstände}

Bedeutsam für das Geschäftssprachegesetz sind insbesondere die politischen und gesellschaftlichen Umstände in den Ostgebieten. Das Königreich Preußen hatte sein Gebiet mit den drei polnischen Teilungen 1772, 1793 und 1795, bei denen Polen zerschlagen und Gebiete annektiert wurden, stetig gen Osten verbreitert. Im Rahmen der territorialen Neuordnung Mittelosteuropas durch den Wiener Kongress 1814/15 blieben Preußen die Gebiete Posen und Westpreußen. ${ }^{79}$ In diesen Gebieten war auch 1815 die Bevölkerung zu großen Teilen polnisch. Auch deswegen proklamierte der preußische König Friedrich Wilhelm III. am 15. Mai 1815 noch folgendes gegenüber seinen polnischen Untertanen in Posen:

„Ihr werdet meiner Monarchie einverleibt, ohne eure Nationalität verleugnen zu dürfen (...) Eure Sprache soll neben der deutschen in allen öffentlichen Verhandlungen gebraucht werden und jedem unter Euch soll nach Maßgabe seiner Fähigkeiten der Zutritt zu allen öffentlichen Ämtern des Großherzogtums (...) offenstehen." ${ }^{\circ 0}$

Für die erste Hälfte des 19. Jahrhunderts kann also noch von einer begrenzten aufklärerischen Toleranz gesprochen werden, die sich erst mit dem nationalistischen Einheitsstreben des Kaiserreichs zu einer harten Sprachunterdrückung mit allen Mitteln wandelte. ${ }^{81}$

79 Eddie/Kouschil, Preußens Polenpolitik am Vorabend des Ersten Weltkrieges, 2018, S. 7.

80 Zitiert nach Cromer, Die Sprachenrechte der Polen in Preußen in der ersten Hälfte des neunzehnten Jahrhunderts, in: Nation und Staat, Bd. 6, 1932/1933, S. 610 (615).

81 von Polenz, Deutsche Sprachgeschichte vom Spätmittelalter bis zur Gegenwart, Bd. III, 1999, S. 110. 


\section{b) Sprache und Nationalismus}

Seit der zweiten Hälfte des 19. Jahrhunderts wurde nicht nur in Deutschland vertreten, dass die Verwirklichung des Nationalstaats eine ihm zugrundeliegende einheitliche Sprache voraussetze. ${ }^{82}$ Mit der nationalistischen Wiederentdeckung der Sprachphilosophie Johann Gottfried Herders und ihrer symbiotischen Verbindung von Sprache und Nation, erschien die Tatsache, dass die Polen in den Ostgebieten weiterhin ihre Sprache pflegten nun staatsgefährdend. ${ }^{83}$ Letztlich befürchtete man, die polnische Sprache verhindere die angestrebte Einheit des Deutschen Kaiserreichs. ${ }^{84}$ Man hing der Vorstellung nach, dass, um den entstandenen polnischen Widerstand gegen die Zerschlagung und Annexion weitestgehend aufzulösen, müsse man nur die polnische Sprache, in der der Geist dieses Volkes wohne, ausmerzen. Dies bewog den Hegemon Preußen zu einer antipolnischen und sprachnationalistischen Germanisierungspolitik, die das langfristige Ziel anstrebte, die nichtdeutschen Sprachen ganz auszulöschen.

\section{c) Sprachenpolitische Germanisierung}

Ausweislich umfangreicher Materialien war das Geschäftssprachegesetz Teil der Germanisierungspolitik des Deutschen Kaiserreichs. ${ }^{85}$ Die sprachpolitische Germanisierung der Ostgebiete setzte an mehreren Stellen an und vollzog sich schrittweise. ${ }^{86}$ So wurde bereits am 14. April 1832 das „Regulativ über die Geschäftssprache der Administrationsbehörden in Posen " erlassen. ${ }^{87}$ Dieses schrieb Deutsch zwingend als innere Amtssprache vor, während Polnisch nur für die mündliche Außensprache zugelassen war. Diese Regelung führte auch dazu, dass einsprachige polnische Beamte keinen Zugang mehr zu Beamtenpositionen hatten. ${ }^{88}$

82 Volkmann, Die Polenpolitik des Kaiserreichs - Prolog zum Zeitalter der Weltkriege, S. 75; Glück, Die preußisch-polnische Sprachenpolitik, 1979, S. 134.

83 von Polenz, Sprachgeschichte Bd. II (Fn. 48), S. 359.

84 Glück, Sprachenpolitik (Fn. 82), S. 129.

85 Ausführliche Materialsammlung zum Geschäftssprachegesetz: Schieder, Das Deutsche Kaiserreich von 1871 als Nationalstaat, 1961, S. $95 \mathrm{ff}$.

86 Zur Sprachenpolitik im gesamten Verlauf: Glück, Sprachenpolitik (Fn. 82), S. 193-340.

87 Glück, Sprachenpolitik (Fn. 82), S. 213 f.

88 Gessinger, Sprachenpolitik gegenüber fremdsprachiger Bevölkerung in Preußen im 19. Jahrhundert, in: Wimmer (Hrsg.), Das 19. Jahrhundert Sprachgeschichtliche Wurzeln des heutigen Deutsch, 1991, S. 106 (116). 
Die Germanisierung setzte ebenso am Schulsystem an. Den kollektiven Sprachenwechsel erhoffte man von hieraus besonders gut durchsetzen zu können, weswegen schrittweise Deutsch als Unterrichtssprache eingeführt wurde. ${ }^{89}$ Als gegen diese Sprachenpolitik weitere polnische Proteste folgten, radikalisierte sich die Germanisierungspolitik in der zweiten Hälfte des 19. Jahrhunderts. ${ }^{90}$

\section{d) Die Begründung des Geschäftssprachegesetzes}

Die Dokumentationen über ein Ansinnen über ein Geschäftssprachegesetz gehen bis in das Jahr 1870 zurück. In einem Bericht des Oberpräsidenten von Posen, Wilhelm Graf von Königsmarck an den Preußischen Staatsminister August Graf zu Eulenburg vom 18. September 1870, spiegelt ersterer die Illoyalität der polnischen Bevölkerung gegenüber dem Preußentum wider und sieht vor "die Provinz Posen in ihrer Totalität (...) zu assimilieren“.91 „Als Haupthinderniß für Erfüllung dieser Aufgabe stellt sich (...) die gesetzlich feststehende Gleichberechtigung der polnischen und deutschen Sprache im öffentlichen Leben dar", stellt Graf von Königsmarck fest und schlägt die Festlegung der deutschen Geschäftssprache vor. ${ }^{92}$ In einem weiteren Bericht des Grafen von Königsmarck vom 20. Februar $1872^{93}$ favorisierte man schließlich ein allgemeines Gesetz, dass das ganze Reich umfassen sollte vor einem nur die Provinz Posen betreffendes, auch um den Anschein eines Polen-Gesetzes zu vermeiden. ${ }^{94}$ Schließlich heißt es in einem Vermerk, der vom Geheimen Oberjustizrat Hermann von Schelling stammt und als eine Art Gesetzesbegründung die Motivlage des Gesetzes widergibt:

„Demgemäß erscheint es auch, insbesondere im Hinblick auf den Umstand, daß die in Preußen herrschende Sprachverschiedenheit zur Schwächung seiner Kraft und Einheit ausgebeutet worden ist, erforderlich, das staatsrechtlich begründete und politisch nothwendige Postu-

89 Broszat, Zweihundert Jahre deutsche Polenpolitik, 1972, S. $134 \mathrm{ff}$.

90 von Polenz, Sprachgeschichte Bd. II (Fn. 48), S. 129.

91 Abgedruckt in Schieder, Nationalstaat (Fn. 90), S. 98 ff.

92 Schieder, Nationalstaat (Fn. 90), S. $98 \mathrm{ff}$.

93 Ebenfalls abgedruckt in Schieder, Nationalstaat (Fn. 90), S. $103 \mathrm{ff}$.

94 Pabst, Das preussische Geschäftssprachegesetz von 1876 - Sprachwechsel nationaler Minderheiten als Mittel politischer Integration, in: Nelde (Hrsg.), Sprachkontakt und Sprachkonflikt, Zeitschrift für Dialektologie und Linguistik, Beihefte, Heft 32, 1980, S. 191 (194). 
lat, daß die Deutsche Sprache als die ausschließliche Geschäftssprache

(...) anzusehen ist, zur Anerkennung zu bringen (...).“95

Die reichsnationalistische Germanisierungspolitik war damit jedoch nicht abgeschlossen. Das Geschäftssprachegesetz läutete sozusagen die zweite Phase nationalistischer Kampfpolitik gegenüber der polnischen Bevölkerung ein. Es folgten zunächst Ausweisungen ${ }^{96}$ und dann eine systematische Bevölkerungs- und Bodenpolitik, die Polen verdrängte und enteignete, um Deutsche anzusiedeln. ${ }^{97}$ Die Germanisierung der Sprache war zu einer Germanisierung des Bodens geworden. Das Endziel der Germanisierung war also die totale Unterdrückung bis hin zur Auslöschung der nationalen Minderheiten. ${ }^{98}$

Das Herstellen einer Traditionslinie zwischen dem Geschäftssprachegesetz, das am 28. August 1876 verkündet wurde ${ }^{99}$ und der derzeitigen Rechtslage scheint also fraglich, wenigstens sind bei einem Verweis auf dieses Gesetz jedoch die Umstände und seine Regelungsabsicht zu spiegeln. ${ }^{100}$

In einer Gesamtschau lässt sich sagen, dass mit dem Aufkommen des Nationalismus und der modernen Nationalstaatsidee das Nebeneinander mehrerer Sprachen, das einmal typisch für Alteuropa gewesen war, zur schiefbeäugten Anomalie wurde. ${ }^{101}$ Das Geschäftssprachegesetz und die damit verbundene Germanisierung bedeuten eine Kehrtwende in der Sprachenpolitik: systematisch wurde Minderheiten der Zugang zu Recht über Sprache gesetzlich versperrt. Die aufklärerische Idee egalitärer Verständ-

95 Zitiert nach Schieder, Nationalstaat (Fn. 90), S. 111.

96 Göthel, Demokratie und Volkstum - Die Politik gegenüber den nationalen Minderheiten in der Weimarer Republik, 2002, S. 60; von Polenz, Sprachgeschichte Bd. III (Fn. 81), S. 129.

97 Broszat, Polenpolitik (Fn. 88), S. 142 ff.

98 Glück, Sprachenpolitik (Fn. 82), S. 130.

99 Zentralblatt für die gesamte Unterrichts-Verwaltung in Preußen, 1876 (Jg. 18), Nr. 9 S. 36, abrufbar unter https://scripta.bbf.dipf.de/viewer/image/985843438_0 018/514/ (letzter Aufruf am 11.05.2021).

100 Vgl. in aller Kürze die Dokumentation der Wissenschaftlichen Dienste des Bundestages, Staatliche Maßnahmen gegenüber der polnischen Minderheit und den Bevölkerungen in den überseeischen Gebieten des Deutschen Reichs 1871 bis 1918, WD 1 - 3000 - 040/18, abrufbar unter: https:/www.bundestag.de/resour ce/blob/594340/1e751eb9b02b5b4ba65a928ce997dbee/WD-1-040-18-pdf-data.pdf (letzter Aufruf am 11.05.2021).

101 Oeter, Juristische Aspekte der Sprachenpolitik, in: Marti (Hrsg.), Sprachenpolitik in Grenzregionen, 1996, S. 39 (40). 
lichkeit war von einer nationalistischen Überhöhung der deutschen Sprache abgelöst worden.

\section{Weimarer Republik}

Dennoch greift Art. 113 der Weimarer Reichsverfassung (WRV) $\mathbb{} 188$ der Paulskirchenverfassung und den damit verbunden Minderheitenschutz fast wortgleich wieder auf:

„Die fremdsprachigen Volksteile des Reichs dürfen durch die Gesetzgebung und Verwaltung nicht in ihrer freien, volkstümlichen Entwicklung, besonders nicht im Gebrauch ihrer Muttersprache beim Unterricht, sowie bei der inneren Verwaltung und Rechtspflege beeinträchtigt werden.“

Trotzdem wurde der preußische Richtungswechsel in der Weimarer Republik fortgeführt. Im Freistaat Preußen war weiterhin das Geschäftssprachegesetz in Kraft, ${ }^{102}$ im Freistaat Bayern galt $₫ 73$ der Bamberger Verfassung, wonach Geschäfts- und Verhandlungssprache im öffentlichen Dienst die deutsche Sprache war. ${ }^{103}$

Zudem fallen die im Vergleich zu 1849 schwächeren Formulierungen auf. So führt Thomas Göthel im Hinblick auf die Rechtsgewährung für Minderheiten treffend aus: „Die 'volkstümliche Entwicklung' darf 1919 nur noch 'nicht beeinträchtigt werden' gegenüber 'ist (...) gewährleistet' von 1849; sodann ist in der Paulskirchenverfassung noch von der 'Gleichberechtigung' der Sprachen die Rede, während 1919 der 'Gebrauch' der minderheitlichen Muttersprachen nur nicht mehr 'beeinträchtigt werden darf" . 104

Hinzu kam ein von der Paulskirchenverfassung abweichendes Nationsverständnis, das auch eine weitere neue Formulierung ins rechte Licht rückt; so heißt es in der Paulkirchenverfassung noch „die nicht deutsch redenden Volksstämme Deutschlands“, die konstitutiv zum „deutschen Volk“ gezählt wurden, während die Weimarer Reichsverfassung die For-

102 Anschütz, Die Verfassung des deutschen Reichs vom 11. August 1919, 14. Aufl. 1933, Art. 113.

103 von Jan (Hrsg.), Die Verfassungsurkunde des Freistaates Bayern vom 14. August 1919, 1927, \$ 73, erläutert, die Norm kollidiere aber nicht mit Art. 113 WRV, da es in Bayern keine fremdsprachigen Siedlungsgebiete gegeben habe.

104 Göthel, Volkstum (Fn. 96), S. 134. 
mulierung "fremdsprachige Volksteile des Reichs“ und damit immerhin das Wortteil "fremd“ enthält. ${ }^{105}$

Trotz der Anknüpfung an sprachliche Minderheitenrechte gelang es nicht, einen Gegentrend zum Sprachnationalismus des Deutschen Kaiserreichs zu initiieren. ${ }^{106}$ In den Kommentierungen zu Art. 113 WRV wurde diesem seine praktische Bedeutung abgesprochen. ${ }^{107}$ So wurde argumentiert, es könnten keine subjektiv-öffentlichen Rechte begründet werden, ${ }^{108}$ zudem bedürfe es eines Ausführungsgesetzes ohne dessen Erlass Art. 113 WRV bedeutungslos sei. ${ }^{109}$ Auch weil ein solches Ausführungsgesetz nie erlassen wurde, muss sich Art. 113 WRV, trotz seines Potenzials, als programmatisch betrachtet lassen. ${ }^{110}$

\section{Sprachvölkische Schule und Nationalsozialismus}

Noch in der Weimarer Republik kam die sprachvölkische Schule auf. Ähnlich zu den Sprachgesellschaften (III.3.) verbindet auch diese Bewegung, die insbesondere vom Allgemeinen Deutschen Sprachverein getragen wird, Nation, Volk und Sprache zu einer Einheit, was in Konsequenz zur Überhöhung der deutschen Sprache und zur Missachtung von Minderheitensprachen führte. ${ }^{111}$ Ab 1933 nähern sich im wissenschaftlichen

105 Göthel, Volkstum (Fn. 96), S. 133.

106 Kraus, Das Recht der Minderheiten (Materialsammlung), 1927, S. 155: eine Ausnahme bildete das Deutsch-polnische Abkommen über Oberschlesien vom 15. Mai 1922, dort Art. 135, der deutsch und polnisch als Amtssprachen zuließ.

107 Anders Laun, Volk und Nation; Selbstbestimmung; nationale Minderheiten, in: Anschütz/Thoma (Hrsg.), Handbuch des deutschen Staatsrechts, Bd. I, 1930, S. 252: „unmittelbar anwendbares Recht“, zitiert nach Broszka, Die Verfassung des Deutschen Reiches vom 11. August 1919, 1980, S. 8.

108 Giese, Die Verfassung des Deutschen Reiches vom 11. August 1919, Art. 113, dies obwohl Art.113 WRV im Zweiten Hauptteil „Grundrechte und Grundpflichten der Deutschen“ und dort im Ersten Abschnitt über „Die Einzelperson“ befindet.

109 Anschütz, Verfassung 1919 (Fn. 102), Art. 113; Gerber, Minderheitenrecht im Deutschen Reich, 1929, S. 8; Gebhard, Handkommentar zur Verfassung des Deutschen Reichs vom 11. August 1919, 1932, Art. 113.

110 Gebhard, Handkommentar (Fn. 109), Art. 113, S. 253.

111 von Polenz, Sprachgeschichte Bd. III (Fn. 81), S. 277. 
Diskurs zudem die Begriffe Sprache und „Rasse“ über den Volksbegriff einander an ${ }^{112}$

Für die Zeit des Nationalsozialismus lassen sich für Deutschland keine Sprachenregelungen die Amtssprache betreffend finden. Dies verwundert, lässt sich aber möglicherweise bereits darauf zurückführen, dass die Vormachtstellung der deutschen Sprache in der staatlichen Kommunikation völlig unbestritten gewesen sein dürfte.

Was die im Krieg besetzten Gebiete betraf, verfolgte der Nationalsozialismus eine imperialistische Germanisierungspolitik nach preußischem Vorbild. So war die Entpolonisierung Polens, die ebenfalls auf einer deutschen Boden-, Schul- und Sprachpolitik beruhte, auch während des Nationalsozialismus erklärtes Ziel. ${ }^{113}$ In diesem Kontext bemerkenswert ist die im besetzten Luxemburg am 6. August 1940 eingeführte Verordnung über den Gebrauch der deutschen Sprache im Lande Luxemburg. ${ }^{114}$ Deren $\mathbb{1}$ sah vor: „Die Amtssprache ist ausschließlich die deutsche Sprache. (...)“.

\section{Die Ambivalenz der deutschen Amtssprache}

Die Geschichte der Amtssprache offenbart, dass Sprachenrecht einerseits dazu geeignet ist, den Zugang zu Recht erst zu ermöglichen und zu erleichtern, andererseits den Zugang zu Recht erschweren oder gar verhindern kann. So bedeutete die Einführung des Deutschen als Rechtssprache im Zuge der Aufklärung eine wesentliche Voraussetzung für die spätere Entwicklung Deutschlands zu einem Rechtsstaat, der die egalitäre Verständlichkeit der Rechts- und damit auch Amtssprache als charakteristisch verstand. Deutsch als Geschäftssprache bedeutete 1876 hingegen das Gegenteil und steht im Kontext von Entmachtung und Unterdrückung der polnischen Minderheit. Die durch die Paulskirchenverfassung gewährten sprachlichen Minderheitenrechte vor der inneren Verwaltung kommen einer Erweiterung von Rechten und einem Mehr an Zugang zu Recht gleich. Hingegen gilt die Begründung des $₫ 23$ Abs. 1 VwVfG als „Lex Gastarbeiter" dem Ausschluss einer bestimmten Gruppe vom Zugang zu Recht.

112 Ausführlich hierzu Antipova, Die nationalsozialistische Sprachpolitik im besetzten Weißrussland 1941-1944, 2018, S. 49 ff.; Knobloch, „Volkhafte Sprachforschung", 2005, S. $287 \mathrm{ff}$.

113 Broszat, Nationalsozialistische Polenpolitik 1939-1945, 1961, S. 21.

114 von Polenz, Sprachgeschichte Bd. III (Fn. 81), S. 151. 
Über das brauchbar gemachte Argument, die Staats- oder Amtssprache schütze die deutschsprachige Bevölkerung vor dem Gebrauch einer Fremdsprache ihnen gegenüber, ${ }^{115}$ offenbart sich neben der geschichtlichen Ambivalenz eine gegenwärtige Ebene der Ambivalenz. Die Schutzwirkung der deutschen Amtssprache deutschkundigen Personen gegenüber ist gesetzgeberisch nicht intendiert und im Gesamten abwegig, zeigt aber, dass die deutsche Amtssprache der deutschsprachigen Mehrheit den Zugang zu Recht gewährt, während sie im selben Akt sprachlichen Minderheiten den Zugang verwehrt. Auch wenn eine monolinguale Amtssprachenregelung für Mehrheiten gemacht wäre, wirkte sie sich also faktisch ausschließend auf sprachliche Minderheiten aus. ${ }^{116}$ Dies gilt allerdings bedeutender vice versa: eine an die Minderheit der „Gastarbeiter:innen“ und Ausländer:innen adressierte Regelung wie $₫ 23$ Abs. 1 VwVfG wirkt sich in der oben beschriebenen Weise als sprachenrechtliche Garantie, wenn wohl auch unbeabsichtigt, ebenso verstärkend auf die Rechte der sprachlichen Mehrheit aus. Trotz dessen wird eine stärkere Verknüpfung von Nation und Sprache gefordert, etwa in Form einer (durch das Grundgesetz geschützten) deutschen Staatssprache. ${ }^{117}$

Eine diesem Aufschlag zur Geschichte der deutschen Amtssprache zugrundeliegende Auffassung ist, dass Recht organisch wächst und sich ebenso entwickelt. Ein solches Verständnis der Rechtsgeschichte ermöglicht es, das historische Recht als Mittel zum besseren Verständnis der geltenden Rechtsordnung oder zu ihrer Fortgestaltung heranzuziehen. ${ }^{118}$ Die ambivalente Geschichte der deutschen Amtssprache legt frei, welche Ideen noch heute unser Rechtsverständnis prägen, markiert doch der $\mathrm{Na}$ tionalismus des Deutschen Kaiserreichs und die seitdem fest etablierte Verbindung von Nation und Sprache einen Wendepunkt hin zu einer monolingualen Ausrichtung des Verwaltungsverfahrens. Diese Perspektive

115 So etwa Aden, ZRP 2009, 53 (53), der ausführt: „Angesichts völlig neuer Fragestellungen im modernen Staat besteht Sprachschutz heute nicht in der Abwehr von Fremdwörtern, sondern in der Sicherung der schichtenneutralen Verständlichkeit.“

116 Ähnlich Poggeschi, Language Rights and Duties in the Evolution of Public Law, 2013, S. $11 \mathrm{f}$.

117 Aden, ZRP 2009, 53 (54); Gesetzentwurf der AfD-Fraktion „Gesetz zur Festschreibung der deutschen Sprache als Landessprache“, BT-Drucks. 19/951.

118 Eisenhardt, Deutsche Rechtsgeschichte, 1984, S.1, mit Verweis auf Conrad, Rechtsgeschichte (Fn 6), S. XVIII; Gmür/Roth, Grundriss der deutschen Rechtsgeschichte, 15. Aufl. 2018, Rn. 7. 
auf die Geschichte der deutschen Amtssprache darf dazu anregen, weder die eigene Zeit noch die derzeitigen Gesetze zu verabsolutieren. ${ }^{119}$

Auch wenn seit der Umsetzung der Europäischen Charta der Regionaloder Minderheitensprachen spezielle Amtssprachenregelungen für deutsche Minderheiten gelten, steht es schlecht um jene Minderheiten, die sich erst in der letzten Hälfte des vergangenen Jahrhunderts in Deutschland etablierten. Waren die sprachlichen Herausforderungen zur Zeit der Einführung des $\mathbb{2 3}$ VwVfG von der Migration der „Gastarbeiter:innen“ geprägt, bestehen derzeitig wohl Sprachbarrieren zwischen Verwaltung und geflüchteten Menschen.

Auch wenn eine kritische Auseinandersetzung mit $\$ 23$ Abs. $1 \mathrm{VwVfG}$ bereits vereinzelt anklingt, ${ }^{120}$ bleibt die Perspektive deutschunkundiger Personen im Zusammenhang von Sprache als Zugangsgarant zu Recht bisweilen zu wenig berücksichtigt.

119 Mitteis, Vom Lebenswert der Rechtsgeschichte, 1947, S. 78.

120 Häberle, Verfassungsprinzipien (Fn. 23), S. 62: „Gastarbeitern und erst recht EGMarktbürgern gegenüber erscheint $₫ 23$ merkwürdig ,nationalstaatlich‘, ja anachronistisch."; mit Verweis auf die Vorgängernorm des $₫ 184$ GVG und deren Entstehen in einer Zeit des betonten Nationalismus: Schmitz, in: Stelkens/Bonk/ Sachs VwVfG (Fn. 4), $\$ 23$ Rn. 5. 
